\title{
The effects of stimulus incongruity on the free looking time of adults and children ${ }^{1}$
}

TERRY T. FAW, University of Califormia, Los Angeles, Calif. 90024

Previous studies, using relative measures of looking behavior, have reported an increasing monotonic relationship between stimulus incongruity and looking time. The present experiment was designed to (1) determine if a similar relationship would be observed if an absolute measure of looking time was employed and (2) determine if differences in patterns of looking behavior would be found for adults and children. The results demonstrate the feasibility of employing a "free-looking" procedure in studies of visual selection and clearly demonstrate for both adults and children an increasing monotonic relationship between degrees of incongruity and looking time.

During the past decade a body of literature has ammassed concerning the influence of stimulus incongruity on visual selection. In various studies it has been demonstrated that an incongruous stimulus (e.g., a horse with a man's head) will be sought out and viewed longer than a banal stimulus (Berlyne, 1958; Faw \& Nunnally, 1967,1968 a). These studies have typically employed stimuli that represent only two points on the continuum of incongruity. Obviously the use of such limited data restricts the statements that can be made concerning the functional relationship between the incongruity inherent in a stimulus and its dominance in visual selection.

Recently, a series of studies have employed stimuli that represent four distinct points on a continuum of incongruity (Faw \& Nunnally, 1968a, b; Nunnally, Faw, \& Bashford, 1969). Using this expanded scale of incongruity, it was found that for both adults and children an increasing monotonic relationship existed between the potential of a stimulus to dominate visual selection and the incongruity inherent in that stimulus. The increasing monotonic relationship reported in those studies is not commensurate with numerou: models of visual selection and stimulus preference that propose that Ss seek out stimuli that generate some optimal level of physiological arousal (e.g., Berlyne, 1960), or stimuli that represent some optimal level of cognitive complexity or stimulus change (e.g., Dember \& Earl, 1957). However, the observed relationship is commensurate with a model of visual selection that assumes that, other things being equal, Ss seek out and view a stimulus for the purpose of resolving the informational conflict inherent in that stimulus, i.e., resolve competing possible interpretations of the incongruous figure (Nunnally, Faw, \& Bashford, 1969).

While the results of the aforementioned studies are in keeping with the hypotheses generated by an information-conflict resolution model of visual selection, the studies in each case employed a procedure in which pairs of stimuli were presented to a $S$ and relative viewing times were recorded. If a simple information-conflict resolution model of visual selection is viable, it must predict that results similar to those obtained using relative measures of visual selection will be obtained when absolute measures of visual selection are employed, e.g., when a $S$ is allowed to view individual stimuli for as long as he chooses.

Procedures that provide an absolute measure of looking time have been used effectively in studies concerning the influence of both stimulus familiarization and stimulus complexity on the visual selection of both adults and children (e.g., Leckart, 1966; Cantor \& Cantor, 1964). However, to the best of the author's knowledge, those procedures have not been used with stimuli representing a multipoint continuum of stimulus incongruity. Furthermore, in the typical "free-looking" procedure, Ss are informed that the experimental stimuli are an important part of the experiment. For example, the $S$ may be told that the $E$ is interested in what people like to look at or in the interest value of certain stimuli. Previous studies have demonstrated that patterns of visual selection can be altered markedly by the demand characteristics of an experiment (Faw, Nunnally, \& Ator, 1969). Consequently, experiments employing instructions that emphasize the role of the stimuli in the experiment may provide results that are not easily generalized outside of the experimental laboratory. It was one purpose of the present study to determine the feasibility of employing a procedure that reduces the potential of obtaining results that can be attributed to demand characteristics implied by the experimental procedure, while at the same time providing an absolute measure of looking time.

In addition to demonstrating the usefulness of an experimental procedure, the major purposes of the present study were: (1) using an absolute measure of visual selection to determine if the increasing monotonic relationship between stimulus incongruity and dominance in visual selection would be observed, and (2) to examine potential differences in the patterns of visual selection exhibited by adults and children.

\section{SUBJECTS}

The Ss were 24 college students and 24 children. The children ranged in age from 7 years, 9 months to 10 years, 8 months, with a mean age of 8 years, 11 months.

\section{APPARATUS}

The physical characteristics of the apparatus have been described in detail elsewhere (Faw \& Nunnally, 1967). The apparatus consisted essentially of a looking box with a translucent screen at one end and a goggle-like opening at the other end. Mounted on the side of the box was a $16-\mathrm{mm}$ movie camera. The lens of the camera extended through a hole in the side of the box and was visible to S. In earlier experiments, the camera was used to photograph S's eye movements; however, in the present study it was used only as a stage prop in our attempts to disguise the true purpose of the experiment and thus diminish potential demand characteristics.

The $S$ rested his chin in a chin rest, looked through the goggle-like opening, and viewed stimulus drawings that were projected on the screen at the far end of the box. A stimulus remained on the screen until $S$ pushed a button, which caused the projector to advance to the next stimulus in the series. The changing of the stimulus was recorded on the moving paper tape of an event recorder.

\section{STIMULI}

The fours sets of stimulus materials employed in the present study have been described in detail elsewhere (Nunnally, Faw, \& Bashford, 1969). Each set of stimulus material consisted of a banal stimulus and stimuli representing three additional levels of incongruity. The incongruity of the stimuli had been determined prior to the experiment by having a group of 27 college students and a group of 54 children rank-order the drawings within each set from most familiar (1) to most unusual (4). The mean rankings obtained in this way did not differ for adults and children. A description of one set of stimuli, the mean ranks, and the variance of those ranks for the pictures in that set follows: normal man $(1.00, .00)$; man with short stubby legs and long arms $(2.00, .00)$; man with four arms (3.04, .19); and man with wings and dog's head $(3.96, .19)$. Similar to the mean ranks of the set described, essentially nonoverlapping distributions of ranks were 
obtained for the other three sets of stimulus materials.

Each stimulus drawing was photographed as a $35-\mathrm{mm}$ acromatic slide. The projected image of these drawings on the viewing screen was approximately $21 \times 14$ in.

\section{PROCEDURE}

Each $S$ was seen individually by $E$. It was explained to $S$ that the experiment in which he was participating concerned the effect of eye movements on changes in pupil size. (Questioning revealed that all Ss knew what was meant by the pupil of the eye, and were aware that the pupil changes size.) $\mathrm{S}$ was told that motion pictures would be taken of his eye and that his only task was to sit quietly and not move his head while the motion pictures were being taken. It was further explained that drawings would be projected onto the screen at the far end of the box. The drawings, $\mathbf{S}$ was told, would give him something to look at during the experiment, but were not themselves a part of the experiment. $S$ was told that there were many pictures available to view, that he could look at each picture as long as he chose, and could change to a new picture by simply pushing the button located at the right-hand side of the looking box.

With those instructions E left the room, the movie camera was activated, and $S$ began to view the randomly ordered series of 16 stimulus slides. After viewing the series of slides, $S$ was allowed to remove his head from the viewing box and relax. Subsequently, the same procedure was repeated and $S$ again viewed the series of 16 stimulus drawings. The order of stimulus presentation during this second period was randomly determined for each $\mathrm{S}$, and differed from the first presentation order.

\section{RESULTS}

The viewing time scores for each stimulus slide were employed in a 2 by 4 by 4 by 2 analysis of variance. Factor $A$ represented the age of $S$, Factor B the four sets of stimulus materials, Factor $C$ the four levels of incongruity, and Factor D the two presentation periods. The results of that analysis revealed three significant effects. Adults viewed stimuli longer than did children, $F(1,46)=6.20, p<.01$. Stimuli in general were viewed longer during the first presentation of the series of slides than during the second presentation, $F(1,46)=10.27, p<.01$. Most important, there was a significant effect attributable to the incongruity of the stimuli, $F(3,138)=9.35, \quad p<.01$. The mean looking time in seconds for the four levels of incongruity were: banal (9.35), Level 2 (9.73), Level 3 (10.72), Level 4 (11.37).
Clearly, the relationship between levels of incongruity and looking time is increasing and monotonic. Subsequent Newman-Keuls tests of multiple comparisons indicate that the viewing time for Level 3 stimuli differed from that for Level 1 stimuli, and the viewing time for Level 4 stimuli differed significantly from that for both Level 1 and Level 2 stimuli (all $\mathrm{p}<.01$ ). No other differences were significant.

\section{DISCUSSION}

The results of the present study provide information of both methodological and theoretical importance. The study demonstrated the feasibility of employing a procedure in studies of visual selection that provides an absolute measure of looking time, yet one that reduces potential demand characteristics implied by the instructions typically employed with similar procedures. In addition to providing an absolute measure of looking time, the "free-looking" procedure has certain advantages over procedures that require the presentation of all possible pairs of stimuli in a set of stimuli to be compared. Using a paired-comparison procedure, the number of stimulus presentations becomes impractical when the set of comparative stimuli exceeds six or seven stimuli, e.g., to compare seven stimuli requires the presentation of 21 stimulus pairs. In contrast, using the "free-looking" procedure one need only employ a single presentation of each stimulus. Thus, the procedure employed in the present study greatly reduces practical restrictions on the number of stimuli that potentially can be compared in a study of visual selection.

While the patterns of looking behavior observed in the present study were similar to those observed when paired-comparison procedures are employed one should be hesitant about concluding that a similar correspondence will exist when examining the effects of all stimulus dimensions on patterns of visual selection. The significant effect on total viewing time attributable to age supports the personal observation that in the present study children were highly motivated to engage in the motor activity required to change the slide projector. Consequently, if the stimulus dimension being manipulated in a study of looking time were to have extremely subtle effects on visual selection, those effects might easily be washed out by the dominant tendency for children to simply push buttons. In spite of this potential problem, however, the "free-looking" procedure employed in the present study has proved to be a useful tool for examining patterns of visual selection.

While the methodological aspects of the present study are of interest, the major contribution of the research was to demonstrate clearly that there exists an increasing monotonic relationship between stimulus incongruity and absolute looking time. As in earlier studies, the observed relationship was similar for both adults and children. The increasing monotonic relationship between incongruity and absolute looking time obtained in the present study, coupled with similar results from earlier studies that used relative measures of visual selection, provides additional support for the information-conflict resolution model of visual selection referred to in the introduction.

The obvious criticism of the aforementioned finding is that there may yet be degrees of stimulus incongruity that would result in a nonmonotonic relationship with looking time. This author, along with his associates, has failed in numerous attempts to construct incongruous stimuli that would be rated by either adults or children as clearly more novel than the most incongruous stimuli in the present sets. Also, it defies the imagination to think of stimuli that would be rated as clearly more novel than, for example, a long-legged "cow" with the rear of an airplane and the trunk of an elephant.

\section{REFERENCES}

BERLYNE, D. E. The influence of complexity and novelty in visual figures on orienting responses. Journal of Experimental Psychology, 1958, 55, 289-296.

BERLYNE, D. E. Conflict, arousal and curiosity. New York: McGraw-Hill, 1960.

CANTOR, J. H., \& CANTOR, G. N. Observing behavior in children as a function of stimulus novelty. Child Development, 1964, 35, 119-128.

DEMBER, W. N., \& EARL, R. W. Analysis of exploratory, manipulatory, and curiosity behaviors. Psychological Review, 1957, 64, 91-96.

FAW, T. T., \& NUNNALLY, J. C. The effects on eye movements of complexity, novelty and affective tone. Perception \& Psychophysics, 1967, 15, 189-192.

FAW, T. T., \& NUNNALLY, J. C. The influence of stimulus complexity, novelty, and affective value on children's visual fixations. Journal of Experimental Child Psychology, 1968a, 6, 141-153.

FAW, T. T., \& NUNNALLY, J. C. A new methodology and finding relating to visual stimulus selection. Psychonomic Science, $1968 \mathrm{~b}, 12,47-48$.

FAW, T. T., NUNNALLY, J. C., \& ATOR, N. A. The influence of stimulus uncertainty and experimental instructions on visual selection. Perception \& Psychophysics, 1969, 5, 137-140.

LECKART, B. T. Looking time: The effects of stimulus complexity and familiarity. Perception \& Psychophysics, 1966, 1, 142-144.

NUNNALLY, J. C., FAW, T. T., \& BASHFORD, $M$. B. The effect of degrees of incongruity on visual fixations in children and adults. Joumal 
of Experimental Psychology, 1969, 81, 360-364.

NOTE

1. This research was supported in part by Research Grant 2634 from the University of
California, Los Angeles. Appreciation is expressed to Evelyn Proctor and Margaret Sacks for their help in this study, and to the University Elementary School at UCLA for their cooperation in providing Ss for the research.

\section{On predicting constrained associates from long-term memory}

\author{
ELIZABETH F. LOFTUS, Stanford \\ University, Stanford, Calif. 94305, and \\ JONATHAN L. FREEDMAN, Columbia \\ University, New York, N.Y. 10027
}

Forty $S$ s gave constrained associates to a conceptual category plus an alphabetic letter (e.g., a musical instrument beginning with " $V$ "). The probability of occurrence of each correct response was predicted from Luce's choice axiom on the basis of associative norms collected by Battig \& Montague (1969). For example, the predicted probability of "violin" being given to "musical instrument starting with $V$ " was calculated as the proportion in the norms of "violin" responses to all responses starting with " $V$ " to the category "musical instrument." The correlation of the predicted and empirical proportions was. 91 .

The purpose of this note is to show the applicability of Luce's choice axiom (Luce, 1959) to data obtained in a constrained association task. The task will be described first, then Luce's axiom, then the data testing the axiom.

College Ss were asked to give verbal associations that met two constraints. For the data of interest the constraints were that the associate had to belong to a specified conceptual category and the word had to begin with a specified letter. For example, the constraints might be "name of a musical instrument beginning with "V" and acceptable responses would be "violin" and "viola." Although the original purpose of this experiment concerned response latencies as a function of various characteristics of the constraints, the present report concerns the relative frequencies of the constrained associates and their prediction by Luce's axiom.

Some notation is needed for stating Luce's axiom. Let $\mathrm{C}$ denote the set of all responses in a particular category (e.g., musical instruments) and let $R$ denote a subset of $C$ that includes a particular response, $x$. $R$ could be the set of all responses in $\mathrm{C}$ that begin with a particular letter and $x$ could be a single response beginning with that letter. Further, let $P(x ; R)$ denote the probability that $x$ is $271 / 3,639$ is the proportion of all chosen when the choice is explicitly responses given that are "violin," while restricted to members of the set $R$. For $271 / 422$ is the proportion of Ss who gave example, if $x=$ violin, and $R=$ all musical "violin" as a response. Similarly, the instruments beginning with the letter $\mathrm{V}$, response "viola" was given 59 times. $\mathrm{P}(\mathrm{x} ; \mathrm{R})$ would denote the probability of "Violin" and "viola" are the only musical "violin" as an associate to the cue "musical instruments starting with $\mathrm{V}$ that appeared instrument-V." Let $P(R ; C)$ be the in the norms. If we were to interpret $271 / T$ probability of choosing any element in the and 59/T as true probabilities in the sense set $\mathbf{R}$ when the choice is restricted to of Luce's axiom, one would expect that members of $C$ and let $P(x, C)$ be the the stimulus "musical instrument starting probability of choosing a particular $x$ from with V" would lead to the response C. Given this notation, then Luce's basic "violin" a proportion calculated according axiom states that:

$$
P(x ; C)=P(R ; C) \cdot P(x ; R) . \quad \text { (1) } P(\text { violin; instrument }-V)=\frac{271 / T}{271 / T+59 / T}
$$

An equivalent formulation is

$$
\begin{aligned}
& =271 / 330 \\
& =.82
\end{aligned}
$$$$
P(x ; R)=P(x ; C) / P(R ; C) .
$$ 